\title{
La genèse des crues dans le bassin de la Loire
}

\author{
par D. Duband (SHF)
}

\section{INTRODUCTION}

De nombreux auteurs (Pardé, Geneslay, Dachary...) ont souligné le fait que depuis 130 ans, on n'a pas observé de crues catastrophiques comparables à celles de 1846, 1866 et particulièrement 1856 à l'amont de Blois et Tours que l'on peut qualifier d'au moins centennales. Le bassin a connu au XXème siècle quelques volumineuses crues à Montjean, mais plus de crue subite extrême. Les chroniques des siècles antérieurs au XIXème siècle, depuis le Moyen Age, font état de tels paroxysmes, mais sans que l'on puisse les quantifier (niveau, débit) comme aux XIXème et XXème siècles. En effet depuis 1846, nous disposons de relevés de cotes atteintes par les plus hautes eaux, dans quelques sections de la Loire et d'affluents. Au XIXème siècle des Ingénieurs des Ponts et Chaussées dont M. Sainjon, et ensuite des Ingénieurs de la 4ème Circonscription Electrique, comme M. Barrière, et du SHC dont $\mathrm{M}$. Gazowski, puis maintenant de la DIREN avec M. Rieux, ont calculé, estimé, reconstitué des débits à partir de courbes de tarage et de calculs hydrauliques.

Grâce à leur compétence et à leur passion pour l'hydrométrie ligérienne, nous disposons de données précieuses de débit des crues historiques avec une incertitude plus réduite (5 à $10 \%)$.

En ce qui concerne les précipitations, les observations journalières et mensuelles sont plus tardives et avec une densité modeste par rapport à celle du réseau climatologique actuel. Mais cette information précieuse est en cours d'archivage par Météo France au pas de temps journalier, elle est indispensable pour analyser la répartition spatiale et temporelle des épisodes de pluie, les intensités et comprendre la genèse des principales crues de la Loire et de ses affluents. On peut d'ailleurs souhaiter vivement la réalisation d'une ou plusieurs monographies (météorologique - climatologique - hydrologique) de la séquence de crues gigantesques et dommageables qui ont concerné non seulement la Loire mais aussi le Rhône comme en mai et juin 1856. Car une analyse approfondie de cet événement hydrologique ainsi que d'autres moins importants pour lesquels on dispose de mesures fiables, pourraient aider à prévenir un événement encore plus rare dû à des configurations, une dynamique d'évolution de la situation météorologique sur l'Europe très particulières, et susceptibles de causer d'immenses dégâts par les eaux déchaînées en extrema de pointe et de volume, d'autant plus que la mémoire humaine est courte (fig.1).

A partir des deux plus longues séries (1863-1995) de débits moyens journaliers reconstitués ou mesurés à Blois et Montjean, on peut faire quelques observations préliminaires.

Tout d'abord, on constate une bonne corrélation entre les modules annuels respectifs avec une variabilité interannuelle considérable (tableau 1).

Mais le contraste entre débits extrêmes de crue peut aussi être frappant si l'on compare le bassin complet de la Loire à Montjean aux sous-bassins amont (tableau 2, fig. 2) (le débit instantané estimé est représenté en italique et le débit moyen journalier en gras, en $\mathrm{m}^{3 / 3} \mathrm{~s}$ ).

\begin{tabular}{|l|c|c|}
\hline & Loire à Blois & Loire à Montjean \\
\hline Volume en milliards de $\mathrm{m}^{3}\left(\mathrm{~km}^{3}\right)$ & & \\
année très sèche, 1949 & 3.9 & 8.9 \\
année moyenne & 11.4 & 26.5 \\
année très abondante, 1910 & 23.0 & 61.8 (70\% du Léman) \\
\hline
\end{tabular}

(la corrélation est extrêmement attenuée quand on considère les couples de débits extrêmes ou de volumes de crue.) Le contraste entre débit moyen journaliers extrêmes observés en étiage et en crue est encore plus saisissant :

\begin{tabular}{|c|c|}
\hline Août & Septembre \\
\hline $20 \mathrm{~m}^{3} / \mathrm{s}$ en 1949 & $6000 \mathrm{~m}^{3} / \mathrm{s}$ en 1866 à Blois \\
$50 \mathrm{~m}^{3} / \mathrm{s}$ en 1949 & $6300 \mathrm{~m}^{3} / \mathrm{s}$ en décembre 1910 à Montjean \\
\hline
\end{tabular}

Tableau 1 : Débits de la Loire à Blois lors d'extrema. 
1. Quelques données sur le bassin de la Loire.

Bassin de la Loire

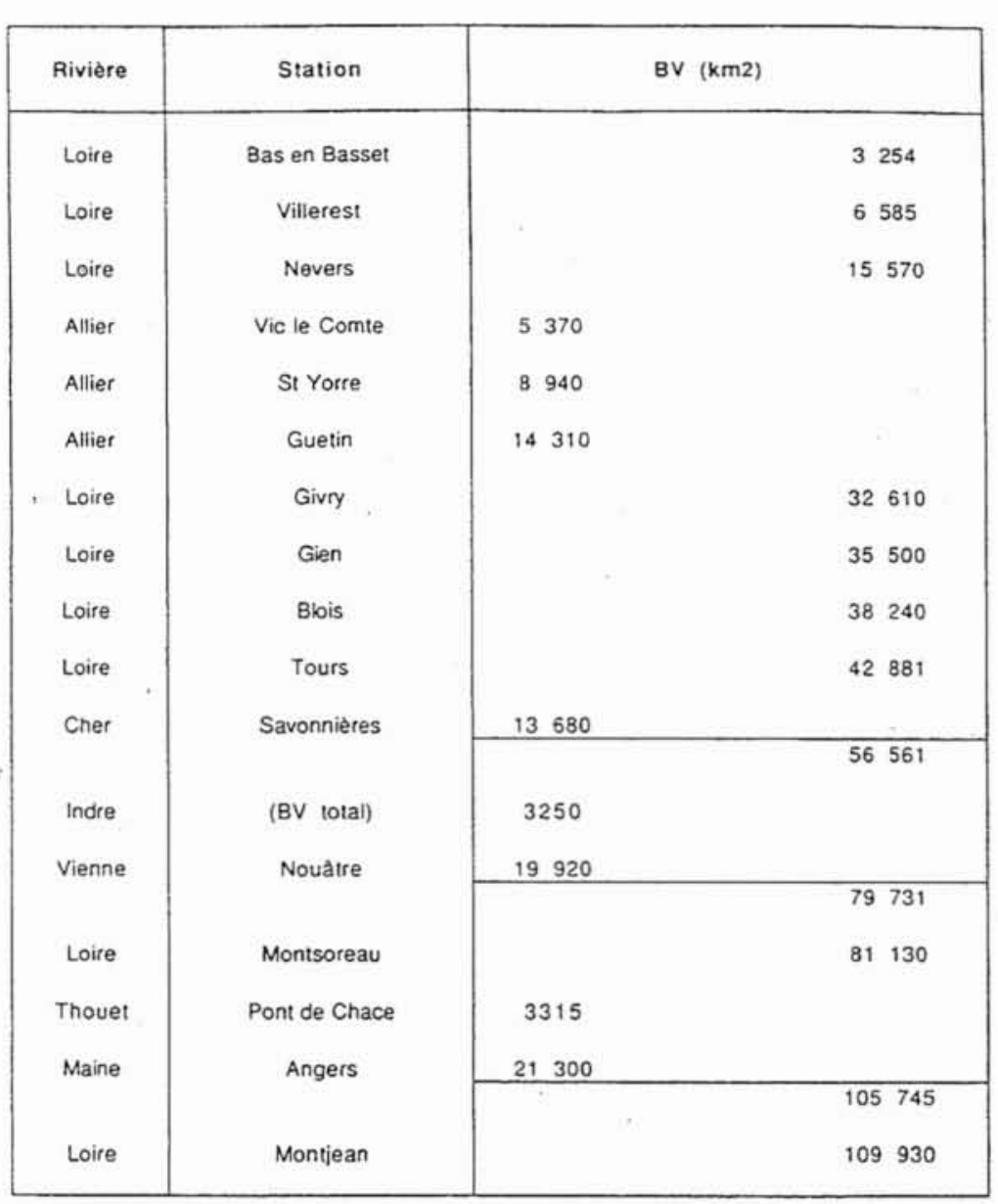

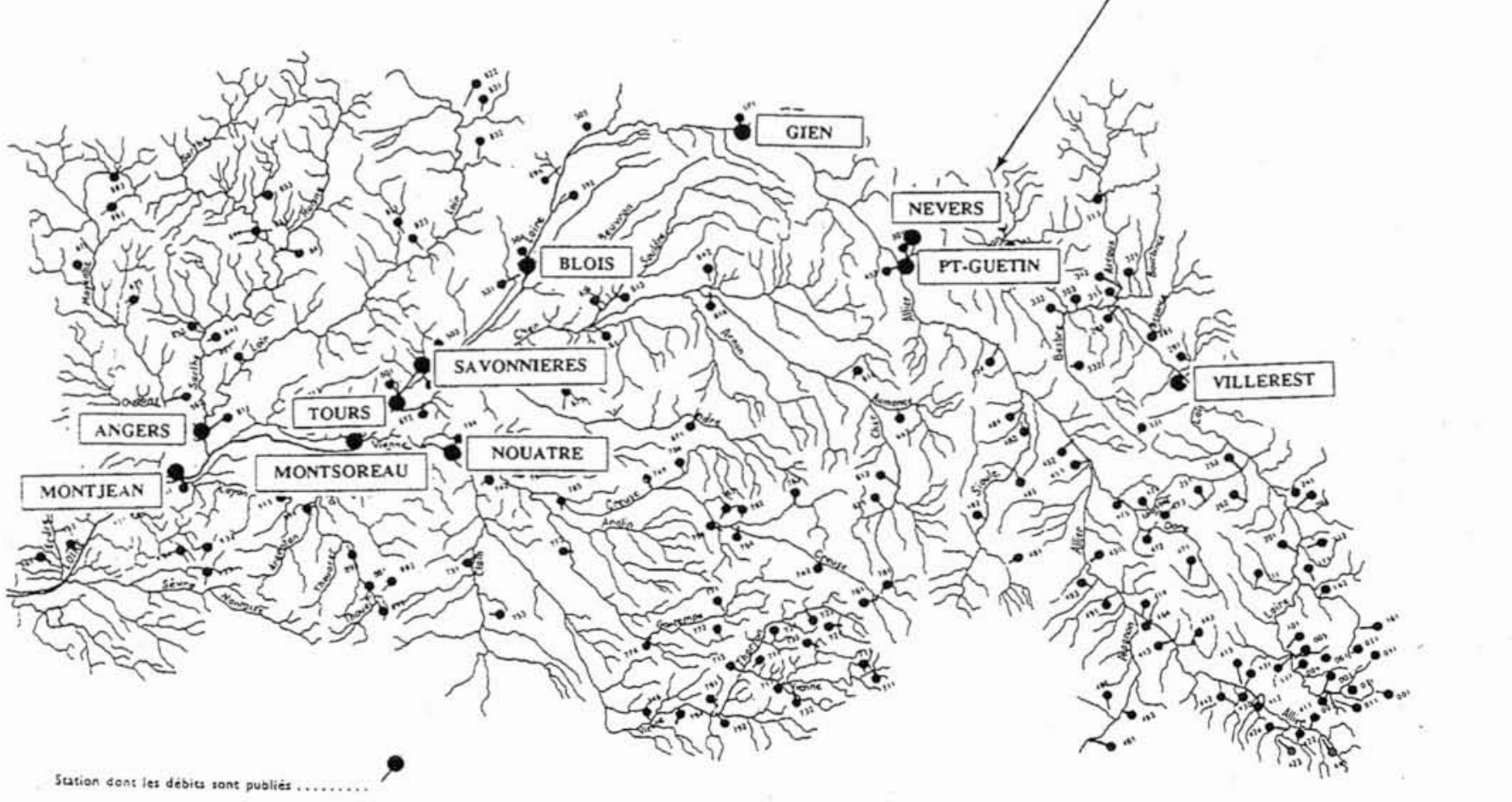


Tableau 2 : Débits de la Loire en différents points du bassin lors de crues.

\begin{tabular}{|l|c|c|c|c|c|c|c|}
\hline \multicolumn{1}{|c|}{ Date } & $\begin{array}{c}\text { VEURDRE } \\
\text { (Allier) }\end{array}$ & $\begin{array}{c}\text { NEVERS } \\
\text { (Loire) }\end{array}$ & $\begin{array}{c}\text { GIVRY } \\
\text { (Loire) }\end{array}$ & $\begin{array}{c}\text { GIEN } \\
\text { (Loire) }\end{array}$ & $\begin{array}{c}\text { BLOIS } \\
\text { (Loire) }\end{array}$ & $\begin{array}{c}\text { TOURS } \\
\text { (Loire) }\end{array}$ & $\begin{array}{c}\text { MONTJEAN } \\
\text { (Loire) }\end{array}$ \\
\hline Oct. 1846 & & & & & & 6000 & 4500 \\
Juin 1856 & 3500 & 4200 & 7600 & 7200 & & 6400 & \\
Sep. 1866 & 3500 & 4500 & 7600 & 7200 & $\mathbf{6 0 0 0}$ & 5300 & $\mathbf{4 9 5 0}$ \\
Oct. 1872 & 1520 & 2770 & 4000 & 3800 & $\mathbf{4 5 0 0}$ & & $\mathbf{3 2 6 0}$ \\
17Sep.1875 & 3060 & 420 & 3250 & 2460 & $\mathbf{1 9 5 0}$ & & $\mathbf{1 3 9 0}$ \\
Oct. 1907 & 1250 & 3270 & 4150 & 3960 & $\mathbf{4 7 0 0}$ & $\mathbf{4 2 0 0}$ & $\mathbf{3 1 2 0}$ \\
Déc. 1910 & 710 & 1370 & 2080 & 2130 & $\mathbf{2 1 0 0}$ & & $\mathbf{6 3 0 0}$ \\
Jan. 1936 & 750 & 1360 & 2110 & 2070 & $\mathbf{2 1 0 0}$ & $\mathbf{2 2 0 0}$ & $\mathbf{5 5 0 0}$ \\
Oct.1943 & 1890 & 2100 & 2970 & 2900 & $\mathbf{2 4 0 0}$ & & $\mathbf{1 7 5 0}$ \\
Déc. 1944 & 1150 & 1920 & 2950 & 3010 & $\mathbf{2 8 0 0}$ & $\mathbf{3 2 0 0}$ & $\mathbf{4 6 7 0}$ \\
Déc. 1982 & 740 & 1560 & 2000 & 2310 & $\mathbf{2 2 8 4}$ & & $\mathbf{6 1 0 0}$ \\
\hline
\end{tabular}

Il y a des épisodes pluvieux localisés qui peuvent engendrer des crues violentes et catastrophiques sur des sous bassins d'affluents de la Loire, de 1000 à $3000 \mathrm{~km}^{2}$ mais, en s'atténuant rapidement vers l'aval, et n'ont que des répercussions très modestes sur la Loire elle-même. Citons en exemples :

- la crue du mai 1940 sur le Cher,

- la crue du 27 octobre 1943 sur la Haute Loire,

- les crues du 3 et 4 octobre 1960 sur le Cher et la Creuse,

- la crue de septembre 1965 sur l'Arroux,

- la crue du 21 septembre 1980 sur la Haute Loire.

\section{II ⿴囗十 CRUES D'ORIGINE MEDITERRANÉ- ENNE EXTENSIVE}

Il s'agit de crues subites et violentes, à très fort gradient, qui se produisent essentiellement de septembre jusqu'au début de décembre, avec quelques sursauts en mai, et qui sont engendrées par un flux de Sud créé par une dépression au Sud de l'Irlande et un anticyclone sur l'Europe Centrale.
Le conflit de masses d'air froid et chaud humide, dont le canal préférentiel est la vallée du Rhône, produit des pluies particulièrement intenses sur les régions cévenoles pouvant déborder jusqu'au Massif Central Est et Nord Est et au Morvan. Elles intéressent la Loire en amont de Nevers, parfois en amont de Villerest, et L'Allier en amont du Veurdre, qui sont les bassins versants privilégiés par les averses intenses (200 à $500 \mathrm{~mm}$ sur les hauts bassins) mais limitées à quelques jours (tableau 3 ). La Loire a très souvent de plus fortes crues que l'Allier, mais la concomittance des crues rares sur les deux bassins peut engendrer depuis le confluent jusqu'à Blois et même Tours des débits instantanés très élevés, d'autant plus dommageables que les crues sont rapides.

On a ainsi atteint plus de $7000 \mathrm{~m}^{3} / \mathrm{s}$ à Givry et Gien en octobre 1846 , septembre 1866 , et plus de $4000 \mathrm{~m}^{3 / \mathrm{s}}$ en octobre 1872 et octobre 1907 et souvent 3000 m³.s.

Pour les crues uniquement d'origine méditerranéenne à l'amont de Gien, les débits entre Blois et Montjean s'atténuent en moyenne de $80 \%$ (fig 3 ).

Ce sont des crues qui transportent un volume d'eau bien moins important (quoique conséquent) que les crues du bassin aval de la Loire.

\begin{tabular}{|l|c|c|c|}
\cline { 2 - 4 } \multicolumn{1}{c|}{} & \multicolumn{3}{c|}{ Pluies mensuelles (mm) } \\
\hline ORLEANS & Sept 1866 & Oct 1872 & Oct 1907 \\
ISSOUDUN & 106 & 106 & 144 \\
LE MANS & 100 & 118 & 138 \\
NANTES & 130 & 140 & 74 \\
EYMOUTIERS & 159 & 96 & 209 \\
ANTULLY & 136 & 202 & 213 \\
DECIZE & - & - & 232 \\
VICHY & 217 & 123 & 278 \\
ROANNE & 240 & 161 & 214 \\
CLERMONT FERRAND & 203 & 146 & 265 \\
LE PUY & 217 & 179 & - \\
JOYEUSE & 227 & - & 463 \\
\hline
\end{tabular}

Tableau 3 : Pluies mensuelles en différents points du bassin de la Loire. 
2. La Loire, crues et embacles (E. GENESLAY)

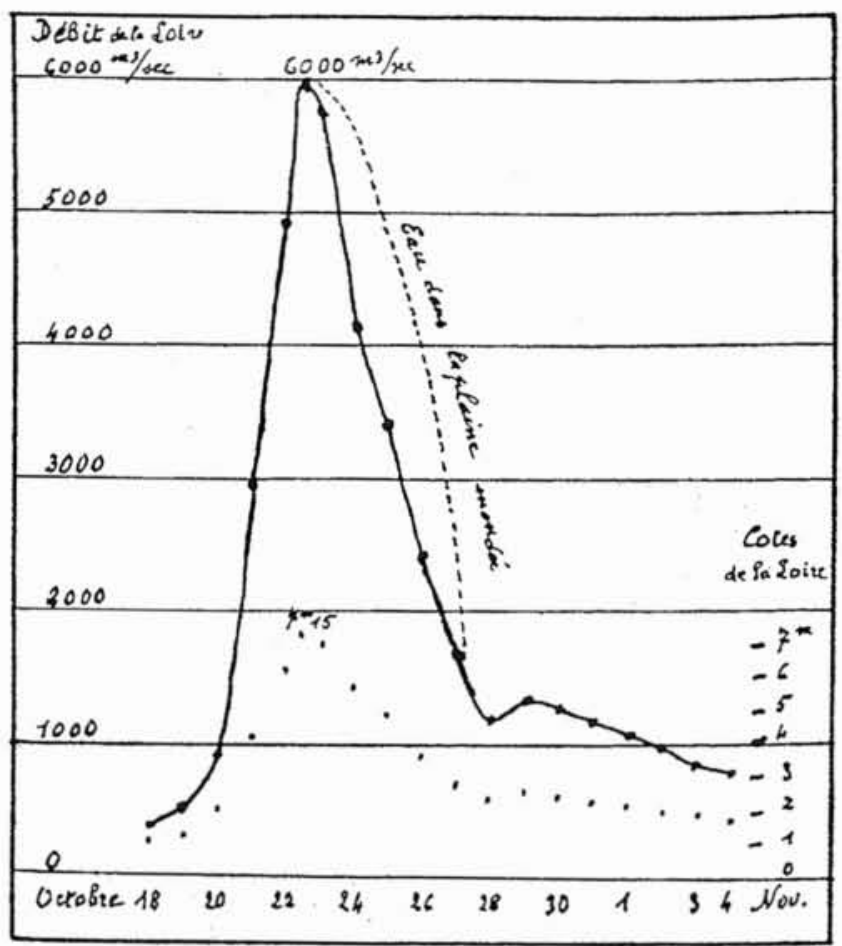

Crue d'octobre 1846

Volume de la crue : $3672000000 \mathrm{de} \mathrm{m}^{3}$ ou $3,762 \mathrm{~km}^{3}$

En trait plein : débit de la Loire en $\mathrm{m}^{3} / \mathrm{s}$

en pointillés : cotes de la Loire à Tours.

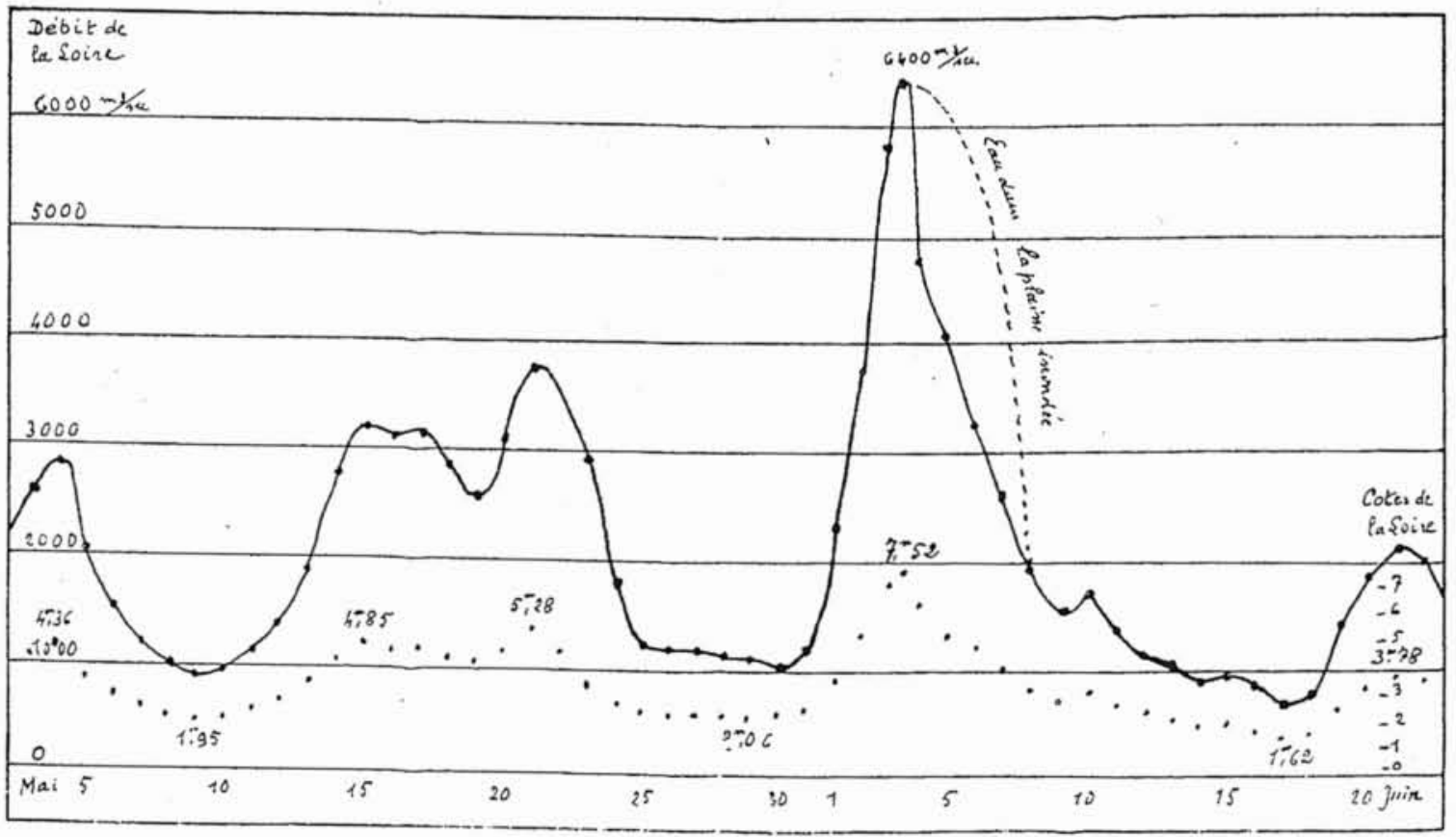

Les crues de la Loire au printemps 1856

Trait plein : débit de la Loire en $\mathrm{m}^{3} / \mathrm{s}$, en pointillés : cotes de la Loire

Volume de la crue : du 1er au 30 mai : $5000400000 \mathrm{~m}^{3}$

du 30 mai au 9 juin : $3315600000 \mathrm{~m}^{3} /$ du 10 au 25 juin : $2052000000 \mathrm{~m}^{3}$

Total : $10368000000 \mathrm{~m} 3$ ou $10,368 \mathrm{~km}^{3}$

Le détail des calculs pour ces crues et les autres se trouve dans les documents remis à la Bibliothèque de la Société de

Géographie de Tours (Bibliothèque municipale). 


\begin{tabular}{|l|c|c|}
\hline \multirow{2}{*}{ Date } & \multicolumn{2}{|c|}{ Volume calculé sur 40 jours pour la Loire à } \\
\cline { 2 - 3 } & BLOIS & MONTJEAN \\
\hline $20 / 09-29 / 10,1866$ & $3.4 \mathrm{~km}^{3}$ & $5.0 \mathrm{~km}^{3}$ \\
$8 / 10-16 / 11,1872$ & $3.0 \mathrm{~km}^{3}$ & $4.2 \mathrm{~km}^{3}$ \\
$10 / 10-18 / 11,1907$ (crue double) & $4.4 \mathrm{~km}^{3}$ & $5.6 \mathrm{~km}^{3}$ \\
\hline \multicolumn{2}{|c|}{$\left(1 \mathrm{~km}^{3}=1 \mathrm{milliard} \mathrm{de} \mathrm{m}^{3}\right)$} \\
\hline
\end{tabular}

Tableau 4 : Volume calculé sur 40 jours à Blois et à Montjean lors de crues.

Dans ces situations le volume de crue à Blois représente environ $70 \%$ du volume de crue à Montjean (tableau 4).

Il y a parfois des précipitations cévenoles qui arrosent aussi bien le versant Est des Cévennes que le versant atlantique, provoquant simultanément sur l'Ardèche, la Loire et l'Allier supérieurs, des crues importantes mais dont les paroxysmes sont rarement concomittants sur l'ensemble des bassins versants.

\section{III 림 CRUES D'ORIGINE OCEANIQUE}

Ce sont habituellement des crues consécutives à une persistance de circulation atmosphérique d'origine atlantique, avec des trains successifs de perturbations pendant un à deux mois consécutifs, et parfois trois (tableau 6).

Ce qui les caractérise est l'important volume d'eau qu'elles propagent en 1 ou 2 mois, ainsi que les débits imposants atteints par montée graduelle.

Les scénarios des quelques grandes crues 1910, 1935-36, 1944, 1982, 1995 présentent des analogies.

En effet la pluie commence à bien humidifier les sols pendant le premier mois, par des averses successives de 3 à 6 jours avec interruption de quelques jours, le même proces- sus recommence le mois suivant et l'on observe alors une succession de crues qui vont se chevaucher en ondulations, et l'une d'entre elles dont la pluie sera particulièrement efficace, de par la saturation progressive d'un grand nombre de surfaces réceptrices, «coiffera» les précédentes (fig. 2).

Le tableau 7 montre l'importance des quantités d'eau qui transitent dans la Loire moyenne et aval :

Le volume de crue en amont de Blois ne représente plus que $35 \%$ en moyenne du volume de crue à Montjean, sauf pour quelques rares crues généralisées où ce pourcentage peut atteindre $50 \%$ (fig 4 ).

On constate que certaines grandes crues dues à des précipitations d'origine océanique peuvent être complétées par une crue résultant de précipitations d'origine méditerranéenne, comme ce fut le cas en novembre-décembre 1910 et également en mai-juin 1856, que Maurice Pardé nomme des «crues générales",

Le fait remarquable est que ces deux dernières crues exceptionnelles furent aussi importantes (avec une prépondérance pour 1856) sur les bassins du Rhône et de la Loire.

Le bassin de la Seine fut surtout concerné par des pluies et une crue marquante en janvier 1910. L'année 1910 représente d'ailleurs des records historiques de pluviométrie cumulée dans une vaste partie de la France.

\begin{tabular}{|c|c|c|c|}
\hline & \multicolumn{3}{|c|}{ Pluies trimestrielles (mm) } \\
\hline & $\begin{array}{c}\text { Avril-Mai-Juin } \\
1856\end{array}$ & $\begin{array}{c}\text { Oct-Nov-Déc } \\
1910\end{array}$ & $\begin{array}{c}\text { Nov-Déc-Janv } \\
1935-36\end{array}$ \\
\hline ORLEANS & 299 & 371 & 253 \\
\hline ISSOUDUN & - & 368 & 259 \\
\hline LE MANS & 163 & 338 & 418 \\
\hline NANTES & 197 & 425 & 464 \\
\hline EYMOUTIERS & - & 609 & 659 \\
\hline ANTULLY & $(460)$ & 508 & 489 \\
\hline DECIZE & 386 & 424 & - \\
\hline VILLENEUVE/ALLIER & 390 & 331 & - \\
\hline VICHY & - & 382 & 238 \\
\hline ROANNE & 421 & 274 & - \\
\hline CLERMONT FERRAND & - & 294 & 123 \\
\hline LE PUY & 385 & 296 & 216 \\
\hline JOYEUSE & - & .761 & 721 \\
\hline
\end{tabular}

Tableau 6 : Pluies trimestrielles en différents points du bassin de la Loire lors de crues d'origine océanique. 


\begin{tabular}{|c|c|c|c|c|c|c|c|c|c|c|c|c|c|}
\hline & \multicolumn{13}{|c|}{ Précipitations mensuelles (en $\mathrm{mm}$ ) } \\
\hline & JAN & FEV & MAR & AVR & MAI & IN & Jut & $A C u$ & $\operatorname{se}$ & $\infty$ & NOV & $\infty E C$ & AN \\
\hline Onleans & 61 & 101 & 58 & 29 & 43 & 48 & 74 & 96 & 106 & 35 & 39 & 60 & 750 \\
\hline Vendóme & 58 & 86 & 49 & 54 & 26 & 34 & 106 & 105 & 70 & 70 & 29 & 40 & 727 \\
\hline Tours & 57 & 97 & 43 & 54 & 59 & 55 & 69 & 75 & 29 & 10 & 21 & 45 & 614 \\
\hline Issoudun & 34 & 95 & 97 & 82 & 71 & 49 & 76 & 108 & 100 & 41 & 51 & 69 & 873 \\
\hline Le Mans & 69 & 77 & 56 & 41 & 89 & 32 & 92 & 101 & 130 & 40 & 33 & 38 & 798 \\
\hline Laval & 105 & 92 & 87 & 53 & 83 & 58 & 92 & 118 & 94 & 24 & 44 & 54 & 904 \\
\hline Nantes & 100 & 112 & 85 & 32 & 60 & 44 & 100 & 111 & 159 & 19 & 35 & 79 & 936 \\
\hline Poitiers & 14 & 116 & 70 & 40 & 65 & 72 & 71 & 54 & 76 & 30 & 47 & 49 & 704 \\
\hline Niort & 94 & 127 & 55 & 78 & 58 & 60 & 103 & 91 & 118 & 32 & 62 & 60 & 938 \\
\hline Eymoutiers & 134 & 155 & 128 & 82 & 76 & 132 & 127 & 121 & 136 & so & 80 & 127 & 1348 \\
\hline \multicolumn{14}{|l|}{ Grosbois. } \\
\hline Pouilly en Auxois & 56 & 95 & 121 & 74 & 71 & 86 & 58 & 98 & 175 & 41 & 67 & 65 & 1007 \\
\hline \multicolumn{14}{|l|}{ Antully } \\
\hline Decize & 43 & 78 & 62 & 71 & 83 & 97 & 105 & 85 & 217 & 36 & 73 & 94 & 1044 \\
\hline Villeneuve/Allier & 25 & 42 & 67 & 72 & 67 & 77 & 75 & 41 & 208 & 36 & 50 & 62 & 822 \\
\hline Vichy & 22 & 44 & 72 & 90 & 77 & 74 & 91 & 126 & 240 & 48 & 41 & 54 & 979 \\
\hline Clermont Ferrand & 27 & 53 & 57 & 41 & 53 & 64 & 51 & 58 & 217 & 41 & 40 & 60 & 762 \\
\hline \multicolumn{14}{|l|}{ Ambert } \\
\hline Le Puy & 19 & 50 & 97 & 88 & 130 & 199 & 57 & 54 & 227 & 50 & 21 & 30 & 1022 \\
\hline Roanne & 20 & 47 & 99 & 77 & 75 & 74 & 66 & 95 & 203 & 16 & 40 & 55 & 867 \\
\hline Joyeuse & & & & & & & & . & & & & & \\
\hline
\end{tabular}

\begin{tabular}{|c|c|c|c|c|c|c|c|c|c|c|c|c|c|}
\hline & \multicolumn{13}{|c|}{ Précipitations mensuelles (en $\mathrm{mm}$ ) } \\
\hline & JAN & FEV & MAR & AVR & MAI & $J u N$ & JUL & $A O U$ & $\operatorname{set}$ & $\infty$ & NOV & $\infty E C$ & AN \\
\hline Orteans & 120 & 94 & 17 & 49 & 110 & 69 & 57 & 70 & 44 & 122 & 166 & 83 & 100 \\
\hline Vendōme & 82 & 79 & 28 & 56 & 74 & 78 & 41 & 62 & 19 & 138 & 161 & 77 & 895 \\
\hline Tours & 101 & 101 & 27 & 61 & 91 & 78 & 58 & 52 & 10 & 118 & 230 & 77 & 100 \\
\hline Issoudun & 114 & 93 & 25 & 51 & 149 & 131 & 57 & 97 & 3 & 111 & 179 & 78 & 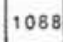 \\
\hline Le Mans & 71 & 99 & 28 & 22 & 52 & 61 & 88 & 23 & 18 & 132 & 146 & 60 & 80 \\
\hline Laval & 103 & 119 & 30 & 27 & 71 & 37 & 90 & 43 & 11 & 84 & 217 & 77 & 90 \\
\hline Nantes & 101 & 161 & 35 & 37 & 55 & 40 & 86 & 100 & 5 & 89 & 226 & 110 & 10 \\
\hline Poitiers & 51 & 112 & 29 & 28 & 92 & 48 & 48 & 63 & 2 & 83 & 164 & 93 & 813 \\
\hline Niont & 92 & 170 & 50 & 47 & 72 & 82 & 59 & 110 & 1 & 114 & 232 & 132 & \\
\hline Eymoutiers & 151 & 221 & 35 & 65 & 217 & 163 & 162 & 91 & 41 & 92 & 331 & 186 & 17 \\
\hline Grosbois & 148 & 140 & 30 & 78 & 157 & 128 & 142 & 107 & 36 & 121 & 160 & 153 & \\
\hline Pouilly en Auxois & 137 & 114 & 22 & 70 & 152 & 100 & 128 & 109 & 30 & 91 & 195 & 149 & 129 \\
\hline Antully & 219 & 141 & 33 & 76 & 153 & 1111 & 145 & 122 & 31 & 116 & 211 & 181 & \\
\hline Decize & 132 & 137 & 21 & 73 & 147 & 201 & 73 & 133 & 34 & 94 & 172 & 158 & 137 \\
\hline Villeneuve/Allier & 81 & 78 & 37 & 49 & 134 & 120 & 89 & 105 & 44 & 74 & 151 & 106 & 10 \\
\hline Vichy & 53 & 106 & 49 & 63 & 150 & 176 & 121 & 108 & 41 & 101 & 140 & 141 & \\
\hline Clermont Ferrand & 45 & 50 & 26 & 51 & 119 & 96 & 86 & 189 & 46 & 89 & 100 & 105 & 100 \\
\hline Amben & 62 & 154 & 34 & 60 & 153 & 95 & 106 & 117 & 31 & 107 & 155 & 107 & \\
\hline Le Puy & 24 & 67 & 67 & 77 & 145 & 78 & 74 & 83 & 19 & 155 & 86 & 55 & 930 \\
\hline Roanne & 45 & 80 & 22 & 54 & 83 & 89 & 122 & 102 & 34 & 100 & 92 & 82 & 905 \\
\hline Joyeuse & 17 & 110 & 60 & 166 & 205 & 43 & 48 & 75 & 29 & 289 & 140 & 332 & 151 \\
\hline
\end{tabular}

Tableau 5 : Précipitations cumulées lors de deux crues (méditerranéenne, océanique) de la Loire à Blois et à Montjean.

Mais seule la crue de 1856 paraît bien représenter la conjonction de situations météorologiques à conséquences catastrophiques, à la fois pour le bassin amont et le bassin aval de la Loire.

\section{IV a ESSAI D'ESTIMATION DES DUREES DE RETOUR DE DEBITS DE CRUE}

La longueur des chroniques historiques de débits extrêmes instantanés et moyens journaliers reconstitués d'après les niveaux d'eau de la Loire à Blois et Montjean autorise à établir la distribution empirique des fréquences cumulées.
Nous avons représenté la distribution du débit moyen journalier maximum annuel pendant 130 ans.

On constate que pour Blois la distribution qui est linéaire dans la partie basse se relève dans sa partie supérieure (patte de chien) où se trouvent essentiellement les crues d'origine méditerranéenne. Nous pensons qu'il ne s'agit pas d'un artefact d'échantillonnage mais bien d'une réalité, et l'application de la méthode de gradex, en s'appuyant sur la distribution les précipitations cumulées en cinq jours extrêmes d'automne, confirmerait cette direction asymptotique.

Le débit moyen journalier décennal est de l'ordre de $2800 \mathrm{~m}^{3} / \mathrm{s}$, alors que le débit centennal peut être estimé à

\begin{tabular}{|l|c|c|c|c|}
\hline \multirow{2}{*}{ Date } & \multicolumn{2}{|c|}{ Volume calculé sur 40 jours à } & \multicolumn{2}{c|}{ Volume calculé sur 2 mois à } \\
\cline { 2 - 5 } & BLOIS & MONTJEAN & BLOIS & MONTJEAN \\
\hline $1 / 11-10 / 12,1910$ & $4.8 \mathrm{~km} 3$ & $13.6 \mathrm{~km}^{3}$ & $7.6 \mathrm{~km}^{3}$ & $20.5 \mathrm{~km}^{3}$ \\
$18 / 12,1935-26 / 1,1936$ & $4.4 . \mathrm{km} 3$ & $12.8 \mathrm{~km}^{3}$ & $6.4 \mathrm{~km}^{3}$ & $18.1 \mathrm{~km}^{3}$ \\
$15 / 11-4 / 11,1944$ & $4.7 \mathrm{~km} 3$ & $9.9 \mathrm{~km}^{3}$ & $6.2 \mathrm{~km}^{3}$ & $12.7 \mathrm{~km}^{3}$ \\
$1 / 12,1982-9 / 1,1983$ & $3.9 \mathrm{~km} 3$ & $10.0 \mathrm{~km}^{3}$ & $4.8 \mathrm{~km}^{3}$ & $12.6 \mathrm{~km}^{3}$ \\
$21 / 01-1 / 3,1995$ & $3.7 \mathrm{~km} 3$ & $12.5 \mathrm{~km}^{3}$ & $4.8 \mathrm{~km}^{3}$ & $15.4 \mathrm{~km}^{3}$ \\
\hline
\end{tabular}

Tableau 7 : Volumes d'eau en Loire moyenne et aval lors de crues d'origine océanique. 




\section{La Loire à Montjean : débit moyen journalier maximal annuel.}

$5000 \mathrm{~m}^{3} / \mathrm{s}$. Ce dernier résultat est cohérent avec les calculs de probabilité des débits extrêmes à Gien réalisés par $\mathrm{Z}$. Gazowski dans son étude (Tableau 8, fig. 4).

Par contre la distribution du débit moyen journalier maximal annuel de la Loire à Montjean, qui est aussi linéaire dans sa partie basse s'abaisse dans la partie haute (patte de cheval) ; s'agit-il d'un effet dû aux débordements dans les champs d'inondation pour les grandes crues au-delà d'un seuil ? De plus on peut considérer que pour les fréquences centennales et supérieures, il y a alors moins de cohérence avec les estimations de crues rares à Gien.

Le débit moyen journalier décennal est de l'ordre de $5200 \mathrm{~m}^{3} / \mathrm{s}$, le débit centennal étant probablement supérieur à $7000 \mathrm{~m}^{3 / 5}$.

\section{$\mathrm{V}$ - CONCLUSION}

Les crues d'origine méditerranéenne sont sans doute moins difficiles à prévoir, car se formant sur les hauts bassins de la Loire et de l'Allier. On y dispose d'un réseau de mesure des intensités des précipitations, bientôt complété par le radar de Sembadel (Chaise Dieu), qui, à l'aide de modèles de relation pluie-débit, permettent d'élaborer des prévisions allant de quelques heures à un jour pour 1000 à $3000 \mathrm{~km}^{2}$.

A l'aval jusqu'à Nevers et le Veurdre, à l'aide de relations de propagation et en tenant compte des apports intermédiaires du Forez et Morvan, qui peuvent être appréciables lors de certaines crues, on dispose d'environ deux jours d'anticipation. Puis entre Givry Fourchambault et Tours, on dispose de deux jours de délai, puis de deux jours de délai supplémentaires entre Tours et Montjean. Tout cela permet de prévoir, grâce à la connaissance des intensités de pluie et leur répartition spatiale, la raideur du front d'onde de crue et la gravité des débits de pointe. Toutefois, la gestion des volumes d'eau de 3 à $4 \mathrm{~km}^{3}$ et plus passant à Givry, Gien, Blois, Tours pendant 4 à 5 jours dès la crue centennale s'avère plus difficile.

Par contre les crues du bassin aval, lentes et volumineuses (entre 5 et $15 \mathrm{~km}^{3}$ d'apports intermédiaires) posent plus de problèmes quant à la prévision des accroissements successifs d'apports en eau, souvent occasionnés par les affluents

\begin{tabular}{|c|c|c|c|}
\hline Durée de retour & NEVERS & LE VEURDRE & $\begin{array}{c}\text { GIVRY } \\
\text { FOURCHAMBAULT }\end{array}$ \\
\hline 10 ans & 2090 & 1200 & 3090 \\
100 ans & 3850 & $3400(3200)^{*}$ & 7150 \\
1000 ans & 6200 & $5360(5600)^{*}$ & 10880 \\
& & *estimation EDF-DTG & \\
\hline
\end{tabular}

Tableau 8 : Estimation des débits instantanés de pointe $\left(\mathrm{m}^{3} / \mathrm{s}\right)$. 
tels que le Cher, l'Indre, la Vienne en rive gauche et le Loir, la Sarthe, la Mayenne en rive droite; la mise en condition de telles crues prend cependant plusieurs semaines sinon quelques mois. Le suivi des précipitations cumulées par un réseau suffisamment dense de stations pluviométriques télétransmises fiables constitue une nécessité, le réseau hydrométrique étant bien étoffé, avec la transmission en temps réel de mesures de débits.

Cependant les volumes d'eau véhiculés par les crues exceptionnelles rendent caduque toute velléité de retenue dans des barrages sur la Loire qui peuvent seulement stocker des crues moyennes (d'ailleurs un véritable barrage écrêteur de crue, avec effet retard, doit être vide en permanence); l'utilisation de zones inondables prévues à cet effet est-elle une solution ? La pire situation pour la moyenne et basse Loire se présentera lorsqu'une violente crue méditerranéenne du bassin amont de Gien se superposera à une crue lente à forts débits alimentée par les affluents aval de la Loire, à la suite de conditions météorologiques particulières, événement rare mais vraisemblable, dont il faut envisager les scénarii.

\section{QUELQUES REFERENCES}

[1] E.GENESLAY - « La Loire - Crues Embacles (1972) * Nouvelles Editions Latines.

[2] A. GOUBET - «Crues exceptionnelles de l'année 1982-83 \#, Société Hydrotechnique de France,

[3] M. PARDE - « Le régime du Rhône »- Etude Hydrologique (1925) Université de LYON - Institut d'Etudes Rhodaniennes : Mélanges PARDE, Institut de Géographie Alpine à GRENOBLE, (1993).

[4] M. GARNIER - " Mémorial de la Météorologie Nationale " Division Climatologie - Longues séries de mesures de précipitations en France, zones 1, 2, 3, 4 (1974),

[5] J.L. ROY - « Protection de la Loire Moyenne contre les inondations",

[6] Z. GAZOWSKI - « Modèles de prévision et de gestion »,

[7] C. PARSY - « Réseau automatique de télémesures hydrométéorologiques du bassin de la Loire ", Revue « Les Travaux Publics " - Inondations, $\mathrm{n}^{\circ} 1036$

[8] Z. GAZOWSKI - «Crues au Bec d'Allier - Reconstitution de débits de crues anciennes à GIVRY, NEVERS, LE VEURDRE »Service Hydrologique Centralisateur du Bassin de Loire Bretagne (1986). 